\title{
Ovalóides em espaço de parâmetros
}

\author{
Ovaloids in parameter space
}

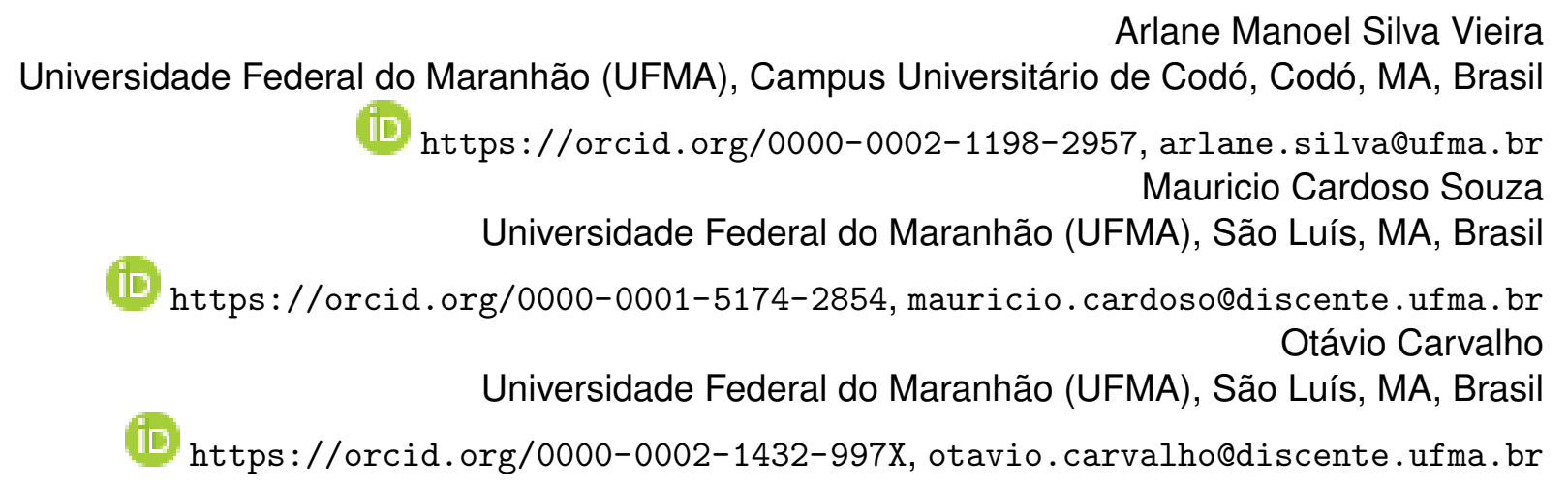

\section{Informações do Artigo}

\section{Como citar este artigo}

VIEIRA, Arlane Manoel Silva; SOUZA, Mauricio Cardoso;

\section{Resumo}

CARVALHO, Otávio. Ovalóides em A dinâmica topológica induzida por inversões geométricas no plano comespaço de parâmetros. REMAT: Revista Eletrônica da plexo já foi abordada e os resultados foram apresentados por Vieira et al. Matemática, Bento Gonçalves, RS, v. 7, n. 1, p. e3007, 12 mar. 2021. DOI: https://doi.org/10. 35819/remat2021v7i1id4191. (2017). Posteriormente, foi verificado que, para uma coleção com três inversões, o espaço de parâmetros das medidas de Markov com suporte no atrator do sistema é um subconjunto aberto de $\mathbb{R}^{3}$ folheado por superfícies de nível compactas definidas pela entropia métrica: superfícies isentrópicas (VIEIRA et al., 2018). O objetivo deste artigo é usar a Teoria de Morse para descrever a geometria global dessas superfícies. Como funções dos níveis, provamos que a área e o diâmetro tendem a zero e que a curvatura Gaussiana é ilimitada, quando os níveis se aproximam do nível crítico (entropia máxima). Em particular, demonstramos que existe

\section{Histórico do Artigo}

Submissão: 13 de maio de 2020. um intervalo aberto maximal de níveis para os quais as superfícies são

Aceite: 4 de dezembro de 2020. ovalóides.

\section{Palavras-chave}

Geometria Global

Curvatura Gaussiana

Espaço de Parâmetros

Teoria de Morse 


\section{Keywords}

Global Geometry

Gaussian Curvature

Parameter Space

Morse Theory

\begin{abstract}
The topological dynamics induced by geometric inversions in the complex plane has already been addressed and the results were presented by $\mathrm{Vi}$ eira et al. (2017). Subsequently, it was proved that, for a set of three inversions, the parameter space of the Markov measure supported by the attractor of the system is an open subset in $\mathbb{R}^{3}$ clad by compact level surfaces defined by metric entropy: isentropic surfaces (VIEIRA et al., 2018). The purpose of this article is to use Morse Theory to describe the global geometry of those surfaces. As functions of the levels, we proved that the area and diameter tend to zero and that the Gaussian curvature is unbounded, when the levels approach the critical level (maximum entropy). In particular, we demonstrated that there is a maximal open range of levels for which the surfaces are ovaloid.
\end{abstract}

\section{Introdução}

Considere três discos fechados $D_{1}, D_{2}$ e $D_{3}$ no plano complexo, dois a dois disjuntos. A fronteira geométrica (círculo) de $D_{j}$ será denotada por $\Gamma_{j}$. A geométrica em $\Gamma_{j}$ é a função definida por

$$
I_{j}(z):=z_{j}+\frac{r^{2}}{\bar{z}-\overline{z_{j}}}
$$

onde $r>0$ é o raio e $z_{j}$ é o centro do disco $D_{j}$.

Com a métrica induzida do plano complexo, $X=D_{1} \cup D_{2} \cup D_{3}$ é um espaço compacto e completo, no qual as inversões $I_{1}, I_{2}$ e $I_{3}$ induzem um sistema dinâmico natural da seguinte forma: uma órbita em $X$ é uma sequência $\left\langle x_{n}\right\rangle \in X^{\mathbb{N}}$, com $x_{j} \in X$ e tal que $x_{j+1}=I_{i}\left(x_{j}\right)$ para algum $i \in\{1,2,3\}$ e $i \neq j$. Esta condição é necessária para garantir que $x_{n} \in X$ para todo $n \in \mathbb{N}$. A motivação principal para esta definição de órbita é a notação de pareamento de círculos introduzida por Mumford, Series e Wright (2002).

O espaço compacto de todas as órbitas em $X$, utilizando a métrica produto relativa de $X^{\mathbb{N}}$, será aqui denotado por $\mathcal{O}$. No entanto, observando-se que uma sequência constante em $X^{\mathbb{N}}$ não corresponde a uma órbita, concluímos que $\mathcal{O}(X)$ é um subespaço próprio de $X^{\mathbb{N}}$.

Além disso, para descrever o conjunto limite das órbitas, podemos associar um itinerário a cada órbita $\left\langle x_{n}\right\rangle$, uma vez que $x_{j} \in D_{s_{j}}$, com $s_{j} \in\{1,2,3\}$, para todo $j \geq 1$, ou seja, $\left\langle s_{n}\right\rangle \in\{1,2,3\}^{\mathbb{N}}$ é o itinerário de $\left\langle x_{n}\right\rangle$, por definição. O conjunto limite de uma órbita $\left\langle x_{n}\right\rangle$ é o conjunto de todos os pontos de acumulação dessa sequência em $X$. Neste caso, órbitas com o mesmo itinerário possuem o mesmo conjunto limite (VIEIRA et al., 2018). 
Essa restrição nos itinerários impõe uma relação de equivalência no espaço das órbitas. Denotaremos por $\widetilde{O}$ o espaço quociente associado, com a correspondente topologia quociente. Note que $\widetilde{O}$ também é compacto. O representante de $\left\langle x_{n}\right\rangle$ em $\widetilde{O}$ será denotado por $\left[x_{n}\right]$. A aplicação $f$ definida por $f\left(\left[x_{n}\right]\right)=\left[x_{n+1}\right]$ é contínua e induz naturalmente uma dinâmica em $\widetilde{O}$. De acordo com Vieira et al. (2017), $f$ admite uma única medida boreliana $\mu$ com entropia métrica maximal com suporte em $\widetilde{O}$, e que

$$
h_{\mu}(f)=\log 2 .
$$

Em geral (veja Vieira et al. (2018)), para cada $v=(a, b, c) \in \mathcal{Q}$ existe uma única medida de probabilidade boreliana $\mu_{v}$ invariante por $f$ com suporte em $\widetilde{O}$, onde $\mathcal{Q}$ é o interior do cubo unitário $[0,1]^{3} \subset \mathbb{R}^{3}$. A recíproca também é verdadeira e, necessariamente, $\mu_{v}$ é ergódica e misturadora. Usaremos a notação

$$
h_{f}(v):=h_{\mu_{v}}(f)
$$

para indicar a entropia métrica de $f$ em relação à medida $\mu_{v}$ como função do parâmetro $v$. Sejam

$$
g(x, y, z)=y(1-x-z)+x z+2 \quad \text { e } \quad \varphi(t)=t \log (t)
$$

para $t>0$ e $\varphi(0):=0$. Foi demonstrado em Vieira et al. $(2018$, p. 180) que, para todo $(a, b, c) \in \mathcal{Q}$,

$$
\begin{aligned}
-g(a, b, c) h_{f}(a, b, c) & =(b-b c+c)[\varphi(a)+\varphi(1-a)]+ \\
& +(1-c+a c)[\varphi(b)+\varphi(1-b)]+ \\
& +(1-a b)[\varphi(c)+\varphi(1-c)] .
\end{aligned}
$$

Em particular, $(a, b, c) \mapsto h_{f}(a, b, c)$ é infinitamente diferenciável em $\mathcal{Q}$ e sua imagem é o intervalo semiaberto $(0, \log 2]$. Além disso, a equação $h_{f}(a, b, c)=\log 2$ admite uma única solução, a saber $(a, b, c)=\left(\frac{1}{2}, \frac{1}{2}, \frac{1}{2}\right)$ (VIEIRA et al., 2017). Como $g(x, y, z) \geq 2$ para quaisquer $x, y, z \in[0,1]$, a função $(x, y, z) \mapsto h_{f}(x, y, z)$ estende-se continuamente ao bordo de $\mathcal{Q}$. Essa extensão também será denotada por $h_{f}$. Para cada $\lambda \in(0, \log 2)$, seja

$$
S_{\lambda}:=\left\{(a, b, c) \in \mathcal{Q} \mid h_{f}(a, b, c)=\lambda\right\}
$$


Figura 1 - Superfície $S_{\lambda}$, para $\lambda \approx 0,42$.

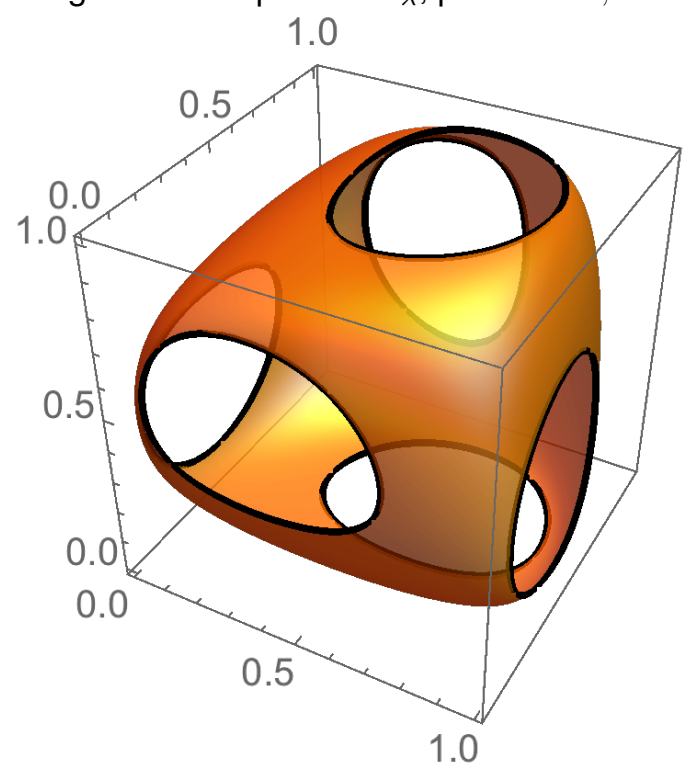

Fonte: Elaboração dos autores (2020).

Para simplificar a notação, usaremos o símbolo $\Phi$ para indicar o número de ouro (MARKOWSKY, 1992), isto é,

$$
\Phi=\frac{1+\sqrt{5}}{2}
$$

O resultado principal deste artigo está resumido no Teorema a seguir.

Teorema 1.1. Para cada $\lambda \in(0, \log 2), S_{\lambda}$ é uma superfície regular compacta. Além disso, $\log \Phi$ é o menor número real positivo para o qual $S_{\lambda}$ é um ovalóide, sempre que $\lambda \in(\log \Phi, \log 2)$.

Um ovalóide é uma superfície compacta e conexa em $\mathbb{R}^{3}$ com curvatura Gaussiana positiva em todos os pontos (DO CARMO, 2010, p. 388). Como os pontos em $S_{\lambda}$ correspondem a medidas invariantes com a mesma entropia métrica, dizemos que $S_{\lambda}$ é uma superfície isentrópica (Figura 1). Uma consequência imediata do Teorema 1.1 é que a curvatura desses ovalóides como função do nível é ilimitada.

Corolário 1.2. A curvatura Gaussiana $K\left(S_{\lambda}\right)$ é ilimitada quando $\lambda \rightarrow \log 2$.

O restante do artigo está organizado da seguinte forma: na Seção 2, apresentamos as superfícies isentrópicas e determinamos o intervalo dos níveis para os quais cada superfície está inteiramente contida em $\mathcal{Q}$; já nas Seções 3 e 4, introduzimos as funções admissíveis e de Morse, e os resultados essenciais desta teoria para a classificação das superfícies isentrópicas; na Seção 5, desenvolvemos resultados preliminares e provamos o Teorema principal, classificando as superfícies 
isentrópicas contidas no cubo $\mathcal{Q}$; finalmente, conclusões e novas direções para a pesquisa são discutidas na Seção 6.

\section{Superfícies isentrópicas}

A função $h_{f}$, definida por (1), é infinitamente diferenciável e possui um único ponto crítico $\left(\frac{1}{2}, \frac{1}{2}, \frac{1}{2}\right)$ com valor crítico igual a $\log 2$. Em VIEIRA et al.(2018), os autores demonstraram que a Hessiana de $h_{f}$ no ponto crítico é

$$
\operatorname{Hess}_{h_{f}}\left(\frac{1}{2}, \frac{1}{2}, \frac{1}{2}\right)=-\frac{4}{3 \ln 10} \operatorname{Id}_{3},
$$

onde $\operatorname{Id}_{3}$ é a matriz identidade de ordem 3. Assim, pelo Teorema da Função Implícita, sempre que $\lambda$ for um valor regular para $h_{f}$, a superfície implícita $S_{\lambda}$ é regular e compacta. Em resumo:

Lema 2.1. Para cada $\lambda \in(0, \log 2)$, a superfície $S_{\lambda}$ é compacta.

Como $h_{f}$ também está definida na fronteira de $\mathcal{Q}$, algumas superfícies $S_{\lambda}$ têm interseção com as faces do cubo $\mathcal{Q}$. Para analisar este contexto, note que

$$
\log (\Phi) \approx 0,481
$$

Proposição 2.2. A superfície $S_{\lambda}$ está contida em $\mathcal{Q}$ se, e somente se, $\lambda \in(\log \Phi, \log 2)$.

Para simplificar a notação, vamos considerar as faces do cubo $\overline{\mathcal{Q}}$ enumeradas de acordo com a Figura 2. O sistema de coordenadas está orientado de forma que as faces 4, 5 e 6 estão contidas nos planos $z=0, y=0$ e $x=0$, respectivamente, e sua interseção é a origem do sistema.

Vamos indicar por $H_{j}$ a restrição da função $h_{f}$ à face $j$ do cubo $\overline{\mathcal{Q}}$. Em particular, a interseção entre a face 4 e a superfície $S_{\lambda}$ é dada pela equação $h_{f}(x, y, 0)=\lambda$, ou seja, $\lambda$ é um nível para a função

$$
H_{4}(x, y)=h_{f}(x, y, 0)=\frac{y(\varphi(x)+\varphi(1-x))+\varphi(y)+\varphi(1-y)}{x y-y-2} .
$$


Figura 2 - Enumeração das faces do cubo $\overline{\mathcal{Q}}$.

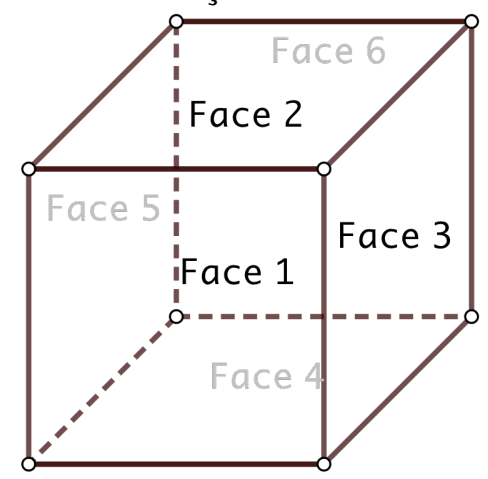

Fonte: Elaboração dos autores (2020).

Nosso objetivo é encontrar um valor para $\lambda$, caso exista, de modo que a equação $H_{4}(x, y)=\lambda$ tenha uma única solução e, ao mesmo tempo, que essa equação não tenha solução para valores maiores de $\lambda$. Uma estratégia para resolver esse problema é estudar $H_{4}$ em relação a máximos e mínimos no quadrado $[0,1]^{2}$.

Primeiro, vamos analisar a função $H_{4}$ na fronteira do quadrado $[0,1]^{2}$, em relação a valores extremos. Usando Cálculo em uma variável, pode-se verificar que, para todo $y \in[0,1]$,

$$
0=H_{4}(0,0)=H_{4}(0,1) \leq H_{4}(0, y) \leq H_{4}\left(0, y_{*}\right) \approx 0,28
$$

onde $y_{*} \approx 0,43$ é a única raíz da equação $(1-y)^{3}=y^{2}$ no intervalo $[0,1]$. $\mathrm{E}$ da mesma forma, para todo $y \in[0,1]$,

$$
0=H_{4}(1,0)=H_{4}(1,1) \leq H_{4}(1, y) \leq H_{4}\left(1, \frac{1}{2}\right)=\frac{\log 2}{2} \approx 0,35
$$

Por outro lado, para todo $x \in[0,1], H_{4}(x, 0)=0 \mathrm{e}$

$$
0=H_{4}(0,1)=H_{4}(1,1) \leq H_{4}(x, 1) \leq H_{4}\left(\frac{1}{2}, 1\right)=\frac{\log 2}{2} \approx 0,35 .
$$

Suponha agora que $(x, y)$ é um extremo relativo de $H_{4}$ no interior do quadrado $[0,1]^{2}$. Então

$$
\frac{\partial H_{4}}{\partial x}(x, y)=\frac{-y[(2+y) \log (x)-2 \log (1-x)+y \log (y)+(1-y) \log (1-y)]}{[y(x-1)-2]^{2}}=0
$$

e

$$
\frac{\partial H_{4}}{\partial y}(x, y)=\frac{-2 x \log (x)-2(1-x) \log (1-x)+(3-x) \log (1-y)-2 \log (y)}{[y(x-1)-2]^{2}}=0 .
$$

Proposição 2.3. Seja $(x, y)$ um ponto crítico de $H_{4}$ no interior do quadrado $[0,1]^{2}$. Então, $x=y$ se, e somente se, $x=\Phi-1$. Em particular, $(\Phi-1, \Phi-1)$ é um ponto crítico de $H_{4}$. 
Demonstração. Seja $(x, y)$ um ponto crítico de $H_{4}$ no interior do quadrado $[0,1]^{2}$ e suponha que $x=y$. De (5) e (6) temos que

$$
\frac{\partial H_{4}}{\partial x}(x, x)=\frac{\partial H_{4}}{\partial y}(x, x)=0 \Leftrightarrow x^{2}+x-1=0 \Leftrightarrow x=\Phi-1 .
$$

Reciprocamente, assuma que $x=\Phi-1$. Então, a condição $\frac{\partial H_{4}}{\partial x}(x, y)=0$ é equivalente a

$$
(1-x)^{-2} x^{y+2}(1-y)^{1-y} y^{y}=1
$$

Por outro lado, a hipótese $x=\Phi-1$ implica que $1-x=x^{2}$. Substituindo em (7), segue que $x^{y-2}(1-y)^{1-y} y^{y}=1$ e, portanto,

$$
\log (x)=\frac{(1-y) \log (1-y)+y \log (y)}{2-y} .
$$

Para determinar o valor de $y$, note que a função

$$
t \mapsto \frac{(1-t) \log (1-t)+t \log (t)}{2-t}
$$

admite extensão contínua ao intervalo $[0,1]$ e é continuamente derivável em $(0,1)$. Assim, pode-se verificar que $t=x$ é o único ponto crítico em $(0,1)$, o que corresponde a um ponto de mínimo global no intervalo $[0,1]$. Além disso, o valor mínimo global é dado por

$$
\frac{(1-x) \log (1-x)+x \log (x)}{2-x}=\frac{(1-x) \log \left(x^{2}\right)+x \log (x)}{2-x}=\log (x) .
$$

Portanto, como o ponto de mínimo global é único, concluímos que $y=x$.

Corolário 2.4. A função $H_{4}$ assume valor máximo relativo no ponto $(\Phi-1, \Phi-1)$, igual a $\log \Phi$.

Demonstração. Com um cálculo direto, segue de (5) que

$$
\frac{\partial^{2} H_{4}}{\partial x^{2}}(x, x)=\frac{-(x-2)^{2}(x+1)-2 x^{2}(x-1) \log (1-x)+4 x^{2}(x-1) \log (x)}{(x-2)^{3}(x-1)(x+1)^{2}},
$$

para todo $x \in(0,1)$. Para $x=\Phi-1$ temos que $1-x=x^{2} \mathrm{e}$, portanto,

$$
\frac{\partial^{2} H_{4}}{\partial x^{2}}(x, x)=\frac{-(x-2)^{2}(x+1)}{(x-2)^{3}(x-1)(x+1)^{2}},
$$

ou seja,

$$
\frac{\partial^{2} H_{4}}{\partial x^{2}}(\Phi-1, \Phi-1)=-\frac{5+\sqrt{5}}{10}<0
$$

Por outro lado, o determinante da matriz Hessiana de $H_{4}$ em um ponto $(x, y)$ no interior do quadrado $[0,1]^{2}$ é dado por:

$$
\operatorname{det}\left(\operatorname{Hess}_{H_{4}}\right)=\frac{\partial^{2} H_{4}}{\partial x^{2}} \cdot \frac{\partial^{2} H_{4}}{\partial y^{2}}-\left(\frac{\partial^{2} H_{4}}{\partial x \partial y}\right)^{2}
$$


Calculando-se as derivadas parciais de segunda ordem de $H_{4}$ diretamente de (5) e (6), para todo $x \in(0,1)$, temos

$$
\begin{aligned}
\operatorname{det}\left(\operatorname{Hess}_{H_{4}}\right)(x, x)= & {\left[(x-2)^{3}(x+1)^{2}+x(x-1)^{2}\left(2+3 x+4 x^{2}\right) \log ^{2}(1-x)-\right.} \\
- & 4 x\left(-2+5 x-5 x^{3}+2 x^{4}\right) \log (x)+4 x(x-1)^{2}\left(2+3 x+4 x^{2}\right) \log ^{2}(x)- \\
- & \left.2 x \log (1-x)\left(2-5 x+5 x^{3}-2 x^{4}+2(x-1)^{2}\left(2+3 x+4 x^{2}\right) \log (x)\right)\right] / \\
& {\left[x(x-1)^{2}(x+1)^{4}(x-2)^{5}\right] . }
\end{aligned}
$$

Em particular, para $x=\Phi-1$, temos

$$
\operatorname{det}\left(\operatorname{Hess}_{H_{4}}\right)=\frac{11+5 \sqrt{5}}{10}>0 .
$$

Pelo critério das derivadas parciais de segunda ordem para extremos de funções de duas variáveis (APOSTOL, 1981, Teorema 9.7), $(\Phi-1, \Phi-1)$ é um ponto de máximo local de $H_{4}$. E ainda,

$$
H_{4}(x, x)=\frac{(1+x)(\varphi(x)+\varphi(1-x))}{x^{2}-x-2}=-\log x=\log \Phi .
$$

De fato, vamos provar a seguir que não existe outro ponto crítico de $H_{4}$ no interior do quadrado $[0,1]^{2}$, de onde concluiremos, em particular, que este ponto crítico é ponto de máximo global de $H_{4}$.

Proposição 2.5. A função $H_{4}$ possui um único ponto crítico no interior do quadrado $[0,1]^{2}$.

Demonstração. Suponha que $(x, y)$ é um ponto crítico de $H_{4}$ no interior do quadrado $[0,1]^{2}$. Então, $x, y \in(0,1)$ e $\nabla H(x, y)=(0,0)$, isto é,

$$
(2+y) \log (x)-2 \log (1-x)+y \log (y)+(1-y) \log (1-y)=0
$$

e

$$
-2 x \log (x)-2(1-x) \log (1-x)-2 \log (y)+(3-x) \log (1-y)=0 .
$$

Pelo Teorema da Função Implícita (RUDIN, 1976, p. 223), a equação (9) define uma função continuamente derivável $x=c_{1}(y)$ implicitamente em $(0,1)$, com extensão contínua ao intervalo $[0,1]$. Neste caso,

$$
c_{1}^{\prime}(y)=\frac{x(1-x) \log \left(\frac{1-y}{x y}\right)}{2+y(1-x)}
$$

para $y \in(0,1)$. Neste intervalo, o único ponto crítico $(x, y)$ da função $c_{1}$ satisfaz a relação $1-y=x y$ que, substituindo-se em (9) obtém-se $y=\Phi-1$ e portanto, $x=\Phi-1$. Vamos provar que esta 
função é crescente no intervalo $[0, \Phi-1]$ e decrescente em $[\Phi-1,1]$. De fato, como $\Phi-1$ é o único ponto crítico no intervalo $(0,1)$, a função $c_{1}^{\prime}$ não muda de sinal em cada um dos intervalos indicados acima. Assim, é suficiente encontrar o sinal da derivada em um ponto interior de cada intervalo. Em particular, substituindo-se $y=\frac{1}{2}$ na equação $\left[9\right.$, obtemos a equação polinomial $x^{5}=4(1-x)^{4}$. Como

$$
x^{5}-4(1-x)^{4}=\left(x^{2}+x-1\right)\left(x^{3}-5 x^{2}+22 x-51\right)
$$

e $x^{3}-5 x^{2}+22 x-51$ não possui raiz real no intervalo $[0,1]$, concluímos que

$$
x=c_{1}\left(\frac{1}{2}\right)=\Phi-1
$$

Logo, para $y=\frac{1}{2}$, temos que

$$
\frac{1-y}{x y}=\Phi>1
$$

e portanto, $c_{1}^{\prime}\left(\frac{1}{2}\right)>0$. Da mesma forma, substituindo-se $y=\frac{3}{4}$ na equação 9 concluímos que

$$
27 x^{11}=256(1-x)^{8}
$$

Essa equação admite uma única raiz real em $[0,1]$, mais precisamente, pelo Teorema do Valor Intermediário (RUDIN, 1976, p. 93), no intervalo $\left[\frac{3}{5}, \frac{4}{5}\right]$. Com isso, para $y=\frac{3}{4}$ e $x \in\left[\frac{3}{5}, \frac{4}{5}\right]$ temos que

$$
0<\frac{1-y}{x y}<1
$$

e, portanto, $c_{1}^{\prime}\left(\frac{3}{4}\right)<0$. Na Figura 3 , é apresentada a curva 1, que é o gráfico de $c_{1}$.

Figura 3 - As curvas 1 e 2 são, respectivamente, os gráficos das equações (9) e 10].

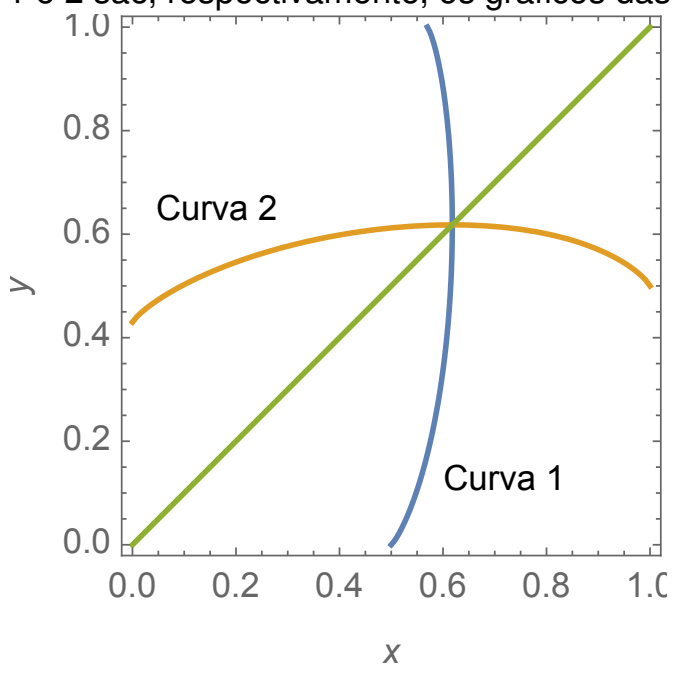

Fonte: Elaboração dos autores (2020). 
Analogamente, a curva 2, indicada na Figura 3 , é o gráfico de uma função $y=c_{2}(x)$ continuamente derivável, definida implicitamente pela equação (10). Neste caso,

$$
c_{2}^{\prime}(x)=\frac{y(1-y)(2 \log (1-x)-2 \log (x)-\log (1-y))}{2+y(1-x)} .
$$

Assim, $c_{2}^{\prime}(x)=0$ se, e somente se,

$$
2 \log (1-x)-2 \log (x)-\log (1-y)=0
$$

ou seja,

$$
y=\frac{2 x-1}{x^{2}} .
$$

Substituindo-se na equação 10 , obtemos

$$
2 \log (1-x)-\log (x)-\log (2 x-1)=0
$$

ou seja, $x=\Phi-1$ e consequentemente, $y=\Phi-1$. A seguir, vamos provar que $c_{2}$ é crescente no intervalo $[0, \Phi-1]$ e decrescente, no intervalo $[\Phi-1,1]$. Inicialmente, observamos que a derivada desta função não muda de sinal em cada um destes intervalos. Assim, para $x_{0}=\frac{1}{2}$ e $y_{0}=y\left(\frac{1}{2}\right)$ temos

$$
2 \log \left(1-x_{0}\right)-2 \log \left(x_{0}\right)-\log \left(1-y_{0}\right)=-\log \left(1-y_{0}\right)>0
$$

e, portanto, $c_{2}^{\prime}\left(\frac{1}{2}\right)>0$. Por outro lado, para $x_{0}=\frac{3}{4}$ e $y_{0}=y\left(\frac{3}{4}\right)$ segue da equação 10 que

$$
2^{16}\left(1-y_{0}\right)^{9}=3^{6} y_{0}^{8}
$$

Pelo Teorema do Valor Intermediário, concluímos que $y_{0} \in\left[\frac{1}{2}, \frac{3}{4}\right]$. Logo,

$$
2 \log \left(1-x_{0}\right)-2 \log \left(x_{0}\right)-\log \left(1-y_{0}\right)=-\log (12)-\log \left(1-y_{0}\right) \leq-\log (3)<0 .
$$

A última desigualdade implica que $c_{2}^{\prime}\left(\frac{3}{4}\right)<0$. Para concluir a demonstração, é necessário verificar que não existe outro ponto crítico de $H_{4}$ no interior de $[0,1]^{2}$. Sejam $a, b \in(0,1)$ e suponha que $(a, b)$ é ponto crítico de $H_{4}$ com $a \neq \Phi-1$, em particular, $a=c_{1}(b)$ e $b=c_{2}(a)$. A primeira condição implica que $a \in\left[\frac{1}{2}, \Phi-1\right]$, uma vez que $c_{1}(0)=\frac{1}{2}$. Segue-se ainda da Proposição 2.3, que $a \neq b$. Então, temos dois casos para considerar, são eles:

CAso I: Assuma que $a<b$. Então

$$
a<b=c_{2}(a) \leq \max c_{2}=\Phi-1 \Rightarrow c_{1}(a)<c_{1}(b) \Rightarrow c_{1}(a)<a .
$$

Por outro lado, verificamos numericamente que $c_{1}^{\prime \prime}(y)<-\frac{2}{5}$ para todo $y \in\left[\frac{1}{2}, \Phi-1\right]$. Em particular, esta condição implica que $c_{1}(a)>a$, uma contradição. 
CASO II: Suponha agora que $a>b$. Pode ocorrer $b<\frac{1}{2}<a$ ou $\frac{1}{2}<b<a$. A primeira desigualdade é impossível já que teríamos

$$
c_{2}(b)<c_{2}\left(\frac{1}{2}\right)<c_{2}(a)=b,
$$

onde $c_{2}\left(\frac{1}{2}\right) \approx 0,612$ é a única raiz da equação $16(1-y)^{5}=y^{4}$ no intervalo $[0,1]$. Quanto à segunda desigualdade, teríamos

$$
c_{2}(b)<c_{2}(a) \Rightarrow c_{2}(b)<b
$$

Analogamente, verificamos numericamente que $c_{2}(x)<-\frac{1}{2}$ para todo $x \in\left[\frac{1}{2}, \Phi-1\right]$ e, portanto, $c_{2}(b)>b$, novamente uma contradição. Assim concluímos a demonstração.

Em relação à restrição de $h_{f}$ às outras faces do cubo $\overline{\mathcal{Q}}$ temos, para quaisquer $x, y \in[0,1]$, $H_{2}(x, y)=H_{4}(y, x)$,

$$
H_{5}(x, y)=H_{4}(1-x, y), \quad \text { e } \quad H_{3}(x, y)=H_{4}(y, 1-x)
$$

E ainda, $H_{6}(x, y)=H_{4}(1-x, 1-y)$ e $H_{1}(x, y)=H_{4}(1-y, 1-x)$. Estas identidades mostram que as curvas de nível das funções $H_{j}$ são iguais, a menos de translações da forma $(x, y) \mapsto(x, y)+(a, b)$ e reflexão em torno da reta $y=x$, ou seja, a aplicação $(x, y) \mapsto(y, x)$ e, reflexão em torno da origem $(x, y) \mapsto(-x,-y)$ (veja a Figura 4). 
Figura 4 - Curvas de nível de cada função $H_{j}$.
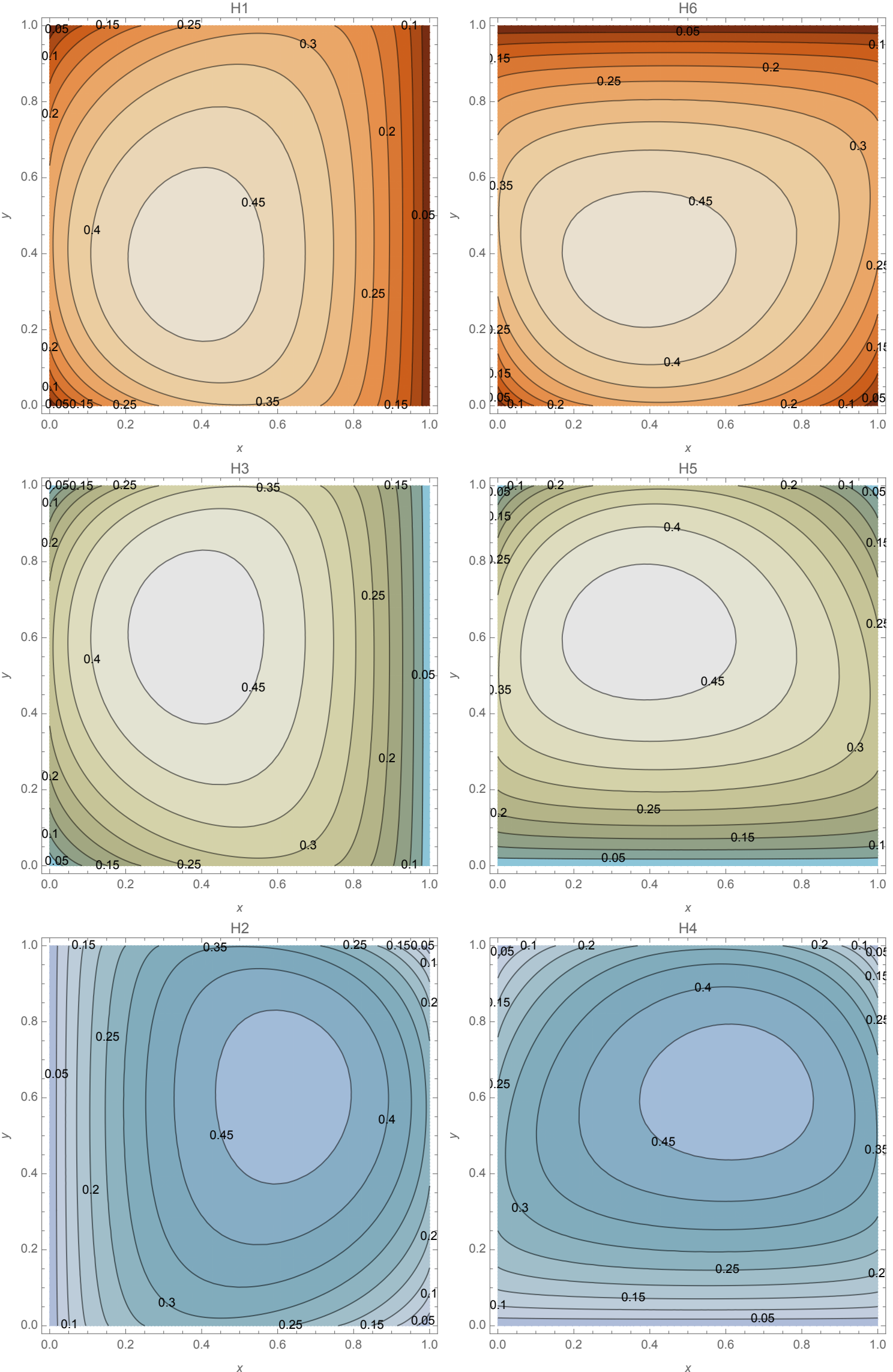

Fonte: Elaboração dos autores (2020). 
Corolário 2.6. Para cada $j=1, \ldots, 6$, a função $H_{j}$ possui um único ponto de máximo global no interior do quadrado $[0,1]^{2}$. Além disso, para cada $j, \log \Phi$ é o valor máximo de $H_{j}$.

\section{Funções admissíveis}

O conceito de funções admissíveis foi introduzido por Leighton (1966) no estudo de estabilidade de Liapunov para funções com um único ponto crítico. Dizemos que uma função diferenciável $F: \mathbb{R}^{n} \rightarrow \mathbb{R}$ tem um ponto crítico no infinito se existe uma sequência $\left\langle x_{n}\right\rangle$ em $\mathbb{R}^{n}$, com $\left\|x_{n}\right\| \rightarrow \infty$ quando $n \rightarrow \infty$, tal que $\left\|\nabla F\left(x_{n}\right)\right\| \rightarrow 0$ quando $n \rightarrow \infty$. Em particular, se $F$ não possui ponto crítico no infinito então existem dois números reais $r>0$ e $\epsilon>0$ tais que $\|\nabla F(x)\| \geq \epsilon$ sempre que $\|x\| \geq r$.

Definição 3.1. Suponha que $F$ é de classe $C^{2}$ em $\mathbb{R}^{n}$. A função $F$ é dita admissível se $F(0)=0$ e $F(x)>0$ próximo da origem.

É uma consequência imediata desta definição que a origem é um ponto de mínimo relativo para qualquer função admissível.

Teorema 3.2 (LEIGHTON, 1966). Seja F uma função admissível sem pontos críticos, finito ou infinito, além da origem. Então, $F(x)>0$ para todo $x \in \mathbb{R}^{n} \backslash\{0\}$. O conjunto de nível $F(x)=c$, onde $c$ é qualquer valor positivo assumido por $F$, é uma variedade $M$ fechada que limita uma região aberta $D$ contendo a origem. Além disso, $F(x)<c$ em $D$ e $F(x)>c$ no exterior de $M$. Finalmente, se $0<c_{1}<c$, a variedade $F(x)=c_{1}$ está inteiramente contida em $D$.

\section{Funções de Morse}

A principal técnica que usaremos para classificar as superfícies isentrópicas é a Teoria de Morse. Seguindo Milnor (1973), apresentaremos alguns resultados fundamentais da teoria nesta seção. Seja $M$ uma variedade de dimensão $n$ e suponha que $F: M \rightarrow \mathbb{R}$ é uma função de classe $C^{2}$. Um ponto crítico de $F$ é um ponto $p \in M$ para o qual a transformação linear $D F_{p}: T_{p} M \rightarrow T_{F(p)} \mathbb{R}$ é nula. Esse ponto crítico é dito não-degenerado se a Hessiana de $F$ em $p$ é uma forma bilinear não-degenerada, ou seja, para todo sistema de coordenadas local $\left(x_{1}, \ldots, x_{n}\right)$ em uma vizinhança de $p$, a matriz Hessiana

$$
\left(\frac{\partial^{2} F}{\partial x_{i} \partial x_{j}}(p)\right)
$$

é não-singular. A dimensão do subespaço de $T_{p} M$ no qual a Hessiana de $F$ em $p$ é definida negativa, é chamada índice de Morse de $F$ em $p$, usualmente denotado por ind $(F, p)$. 
Definição 4.1. A função $F$ é chamada função de Morse se todos os seus pontos críticos são nãodegenerados.

Lema 4.2 (Lema de Morse). Sejam $M \subseteq \mathbb{R}^{n}$ um aberto e $p$, um ponto crítico de uma função de Morse $F: M \rightarrow \mathbb{R}$ de classe $C^{2}$. Então existem uma vizinhança $U$ de $p$, uma vizinhança $V \subseteq \mathbb{R}^{n}$ da origem e um difeomorfismo $\psi: U \rightarrow V$ tais que $\psi(p)=0$ e

$$
\left(F \circ \psi^{-1}\right)\left(x_{1}, \ldots, x_{n}\right)=F(p)-x_{1}^{2}-x_{2}^{2}-\cdots-x_{i}^{2}+x_{i+1}^{2}+\cdots+x_{n}^{2},
$$

para todo $\left(x_{1}, \ldots, x_{n}\right) \in V$, onde $i=\operatorname{ind}(F, p)$.

Para enunciar o Primeiro Teorema de Morse, também chamado Lema de deformação, consideremos os seguintes conjuntos:

$$
M_{a}:=\{x \in M ; F(x) \leq a\} \quad \text { e } \quad M_{[a, b]}:=\{x \in M ; a \leq F(x) \leq b\} .
$$

Teorema 4.3. Seja $F: M \rightarrow \mathbb{R}$ uma função de classe $C^{2}$. Suponha que o conjunto $M_{[a, b]}$ seja compacto e que não contenha pontos críticos de $F$. Então, $M_{a}$ e $M_{b}$ são difeomorfas.

\section{Geometria global}

Considere as translações $T_{1}$ e $T_{2}$ definidas por

$$
T_{1}(x)=x+\log 2 \quad \text { e } \quad T_{2}(x, y, z)=(x, y, z)+\left(\frac{1}{2}, \frac{1}{2}, \frac{1}{2}\right),
$$

para quaisquer $x, y, z \in \mathbb{R}$. Então, o conjunto $\mathcal{Q}_{0}:=T_{2}^{-1}(\mathcal{Q})$ é um cubo aberto de lado unitário com centro na origem. Assim, podemos considerar a função

$$
F(x, y, z):=-\left(T_{1}^{-1} \circ h_{f} \circ T_{2}\right)(x, y, x)
$$

para todo $(x, y, z) \in \mathcal{Q}_{0}$. Logo, $F$ é infinitamente diferenciável e tem as seguintes propriedades: possui um único ponto crítico na origem, $F(0,0,0)=0$ e $F(x, y, z)>0$ para $(x, y, z) \neq(0,0,0)$ em $\mathcal{Q}_{0}$. Portanto, $F$ é uma função admissível.

Dado $\lambda \in(\log \Phi, \log 2)$, denotaremos por $K_{\lambda}$ a região fechada de $\mathbb{R}^{3}$ limitada por $S_{\lambda}$. O resultado a seguir é uma consequência do Teorema 3.2 .

Lema 5.1. Para cada $\lambda \in(\log \Phi, \log 2)$, a superfície $S_{\lambda}$ é fechada e conexa. O compacto $K_{\lambda}$ contém o ponto o crítico de $h_{f}$ em seu interior. Além disso, $h_{f}(x)>\lambda$ no interior de $K_{\lambda}$ e $h_{f}(x)<\lambda$ no exterior de $K_{\lambda}$. Finalmente, se $0<\lambda<\alpha<\log 2$ então $S_{\alpha}$ está inteiramente contida no interior de $K_{\lambda}$. 
Proposição 5.2. Para quaisquer $\alpha, \beta \in(\log \Phi, \log 2)$, as superfícies $S_{\alpha}$ e $S_{\beta}$ são difeomorfas.

Demonstração. Podemos supor sem perda de generalidade que $\alpha<\beta$. Como $S_{\alpha} \cap S_{\beta}=\varnothing$ temos que $K_{\beta} \subset K_{\alpha}$. Seja $M:=K_{\alpha} \backslash \operatorname{int}\left(K_{\beta}\right)$. Então, $M$ é uma variedade fechada diferenciável tridimensional e o intervalo $[\alpha, \beta]=h_{f}(M)$ não contém o valor crítico de $h_{f}$. Como $h_{f}$ é uma função de Morse em $M$, o Teorema 4.3 garante que existe um difeomorfismo $G: K_{\beta} \rightarrow K_{\alpha}$. Portanto, a restrição $\left.G\right|_{S_{\beta}}$ é um difeomorfismo entre $S_{\beta}$ e $S_{\alpha}$.

A matriz hessiana de $h_{f}$ em (2) no ponto crítico tem um único autovalor $-\frac{4}{3 \log 10}$ com multiplicidade 3 e, portanto, o ponto crítico de $h_{f}$ é não-degenerado e possui índice de Morse igual a 3. Pelo Lema de Morse 4.2, existe uma vizinhança $U$ de $\left(\frac{1}{2}, \frac{1}{2}, \frac{1}{2}\right)$ em $\mathcal{Q}$, uma vizinhança $V$ de $(0,0,0)$ em $\mathbb{R}^{3}$ e um sistema de coordenadas locais $\psi: U \rightarrow V$ tais que

$$
\left(h_{f} \circ \psi^{-1}\right)(x, y, z)=\log (2)-x^{2}-y^{2}-z^{2},
$$

para todo $(x, y, z) \in V$. Seja $r>0$ suficientemente pequeno para que a esfera $\mathcal{E}(r)$ com centro na origem e raio $r$ esteja contida em $V$. Denotaremos por $\mathcal{B}(r)$ a bola fechada cuja fronteira é $\mathcal{E}(r)$. Seja $\alpha<\log (2)$ tal que $(\alpha, \log 2) \subset\left(h_{f} \circ \psi^{-1}\right)(\mathcal{B}(r))$. Com isso,

$$
\left(h_{f} \circ \psi^{-1}\right)(x, y, z)=\alpha \Leftrightarrow x^{2}+y^{2}+z^{2}=\log (2)-\alpha,
$$

e, portanto,

$$
\psi\left(S_{\alpha}\right)=\mathcal{E}(\sqrt{\log (2)-\alpha}) .
$$

Isto prova que $S_{\alpha}$ é difeomorfa a uma esfera de raio $\sqrt{\log (2)-\alpha}$.

Proposição 5.3. A superfície $S_{\lambda}$ é difeomorfa a uma esfera. Além disso,

$$
\operatorname{diam}\left(S_{\alpha}\right) \rightarrow 0 \quad \text { quando } \quad \alpha \rightarrow \log 2 .
$$

Demonstração. Seja $\alpha<\log 2$ tal que $S_{\alpha}$ é difeomorfa a $\mathcal{E}(r)$. Pela Proposição $5.2, S_{\lambda}$ é difeomorfa $S_{\alpha}$ e, portanto, $S_{\lambda}$ é difeomorfa a $\mathcal{E}(r)$. Para finalizar a prova, falta verificar que $\operatorname{diam}\left(S_{\alpha}\right) \rightarrow 0$ quando $\alpha \rightarrow \log 2$. Para isto, vamos supor que a conclusão é falsa. Neste caso, existe uma sequência $\left(\alpha_{n}\right)$ no intervalo $(\log \Phi, \log 2), \operatorname{com} \alpha_{n} \rightarrow \log 2$ quando $n \rightarrow \infty$, e um número real $\epsilon>0$ tais que $\operatorname{diam}\left(S_{\alpha_{n}}\right) \geq$ $\epsilon$ para todo $n$. Como $S_{\alpha_{n}}$ é um compacto, existem $a_{n}$ e $b_{n}$ em $S_{\alpha_{n}}$ tais que $\operatorname{diam}\left(S_{\alpha_{n}}\right)=\left\|a_{n}-b_{n}\right\|$ para todo $n \geq 1$. Seja $n_{0} \geq 1$ tal que $\alpha<\alpha_{n}<\log (2)$ para todo $n \geq n_{0}$. Então

$$
2 \sqrt{\log (2)-\alpha_{n}} \geq\left\|\psi\left(a_{n}\right)-\psi\left(b_{n}\right)\right\| \geq\left\|a_{n}-b_{n}\right\| \inf \|D \psi\| \geq \epsilon \inf \|D \psi\|,
$$


para todo $n \geq n_{0}$, onde o ínfimo de $\|D \psi\|$ é tomado sobre o conjunto $\psi^{-1}(\mathcal{B}(\alpha))$. Logo, inf $\|D \psi\|=0$. Lembrando que a aplicação $D \psi: U \rightarrow \mathcal{L}\left(\mathbb{R}^{3}\right)$ é contínua, existe $\left(x_{0}, y_{0}, z_{0}\right) \in \psi^{-1}(\mathcal{B}(\alpha))$ tal que $\left\|D \psi\left(x_{0}, y_{0}, z_{0}\right)\right\|=0$, e isto contradiz o fato de que $\psi$ é um difeomorfismo.

Corolário 5.4. Quando $\alpha \rightarrow \log (2)$, a área de $S_{\alpha}$ tende a 0 .

Demonstração. Suponha que a conclusão é falsa. Então existe uma sequência $\left(\alpha_{n}\right)$ no intervalo $(\log \Phi, \log 2), \operatorname{com} \alpha_{n} \rightarrow \log 2$ quando $n \rightarrow \infty$, e um número real $\epsilon>0$ tais que

$$
\text { área }\left(S_{\alpha_{n}}\right) \geq \epsilon
$$

para todo $n$. Seja $r_{n}:=\sqrt{\log (2)-\alpha_{n}}$. Sem perda de generalidade, suponha que $\left(\alpha_{n}\right)$ é uma sequência crescente. Então existe $n_{0} \geq 1$ tal que $\psi\left(S_{\alpha_{n_{0}}}\right)=\mathcal{E}\left(r_{n_{0}}\right)$. Logo, para $n \geq n_{0}$,

$$
4 \pi r_{n}=\operatorname{área}\left(\mathcal{E}\left(r_{n}\right)\right)=\int_{\mathcal{E}\left(r_{n}\right)} \mathrm{d} A=\int_{\psi\left(S_{\alpha_{n}}\right)} \mathrm{d} A=\int_{S_{\alpha_{n}}}|\operatorname{det}(D \psi)| \mathrm{d} A .
$$

Logo, para todo $n \geq n_{0}$,

$$
4 \pi r_{n} \geq\left(\inf _{S_{\alpha_{n}}}|\operatorname{det}(D \psi)|\right) \text { área }\left(S_{\alpha_{n}}\right) \geq \epsilon \inf _{S_{\alpha_{n}}}|\operatorname{det}(D \psi)| \geq \epsilon \inf _{S_{\alpha_{n_{0}}}}|\operatorname{det}(D \psi)| .
$$

Como $r_{n} \rightarrow 0$ quando $n \rightarrow \infty$, segue-se que

$$
\inf _{S_{\alpha_{n}}}|\operatorname{det}(D \psi)|=0
$$

e, portanto, existe $\left(x_{0}, y_{0}, z_{0}\right) \in S_{\alpha_{n_{0}}}$ tal que $D \psi\left(x_{0}, y_{0}, z_{0}\right)$ não é inversível, uma contradição.

Demonstração do Corolário 1.2. Para $\lambda>\log \Phi, S_{\lambda}$ é uma superfície compacta, conexa e orientável, homeomorfa a uma esfera, e pelo Teorema de Gauss-Bonnet (DO CARMO, 2010, p. 331) obtemos

$$
\int_{S_{\lambda}} K \mathrm{~d} A=2 \pi \chi\left(S_{\lambda}\right)=4 \pi
$$

Assim,

$$
4 \pi \leq \int_{S_{\lambda}}|K| \mathrm{d} A \leq\left(\max _{S_{\lambda}}|K|\right) \cdot \operatorname{área}\left(S_{\lambda}\right)
$$

Como área $\left(S_{\lambda}\right) \rightarrow 0$ quando $\lambda \rightarrow \log (2)$, pelo Corolário 5.4, segue-se que $\lim _{\lambda \rightarrow \log 2}|K|=\infty$.

Demonstração Teorema 1.1. Tendo em vista os Lemas 2.1 e 5.1, e as Proposições 2.2 e 5.3, é suficiente provar que a curvatura Gaussiana é positiva em cada ponto de $S_{\lambda}$, para $\lambda \in(\log \Phi, \log 2)$. Inicialmente vamos provar que que $S_{\lambda}$ não tem pontos planares. Para tanto, seja $\alpha<\log 2$ tal que $\psi\left(S_{\alpha}\right)=\mathcal{E}(r)$, onde $r=\sqrt{\log (2)-\alpha}$. Denotaremos por $N_{\alpha}$ e $N_{r}$ as aplicações de Gauss de $S_{\alpha} \mathrm{e}$ $\mathcal{E}(r)$, respectivamente. Então,

$$
N_{\alpha}=N_{r} \circ \psi
$$


e, pela regra da Cadeia,

$$
\left(D N_{\alpha}\right)_{p}=\left(D N_{r}\right)_{\psi(p)} \circ(D \psi)_{p}
$$

para todo $p \in S_{\alpha}$. Denotando por $K(p)$ a curvatura Gaussiana de $S_{\alpha}$ em $p$, temos

$$
K(p)=\operatorname{det}\left(D N_{\alpha}\right)_{p}=\operatorname{det}\left(D N_{r}\right)_{\psi(p)} \cdot \operatorname{det}(D \psi)_{p}=\frac{1}{r^{2}} \operatorname{det}(D \psi)_{p}
$$

Como $(D \psi)_{p}$ é um isomorfismo linear, segue-se que $K(p) \neq 0$. Em particular, a função $S_{\alpha} \ni p \mapsto$ $K(p)$ não muda de sinal, já que $S_{\alpha}$ é conexa e $K$ é contínua. Por outro lado, como $S_{\alpha}$ é compacta, isso implica que existe ao menos um ponto com curvatura positiva (O'NEILL, 2006, p. 277, DO CARMO, 2010, p. 387), e assim provamos que $K(p)>0$ para todo $p \in S_{\alpha}$. Por fim, sabendo que $S_{\lambda}$ e $S_{\alpha}$ são difeomorfas, usamos a mesma ideia para concluir que todos os pontos de $S_{\lambda}$ são elípticos.

\section{Considerações finais}

Em nosso contexto, a estrutura ergódica associada ao espaço de órbitas depende da medida de Markov invariante que estamos considerando. Cada medida está associada a um parâmetro e vice-versa. Portanto, é fundamental descrever esse espaço de parâmetros sob os aspectos topológico, geométrico, combinatório e mensurável, por exemplo, para entender como essa estrutura muda a dinâmica mensurável.

Neste sentido, o primeiro passo deste roteiro é classificar a geometria dos conjuntos de nível da função entropia métrica em relação aos parâmetros. Considerando apenas três inversões geométricas, verificamos que o espaço de parâmetros associado às medidas de Markov é a região interior do cubo unitário, folheado por superfícies compactas. Mais importante, provamos que o interior desse cubo possui um subconjunto aberto folheado por ovalóides difeomorfos. Nossos resultados indicam ainda que a estrutura geométrica qualitativa (linhas de curvatura, geodésicas, pontos umbílicos, etc.) em quaisquer dois desses ovalóides, é a mesma. Este é o primeiro passo para descrição da geometria do espaço de parâmetros.

Cada ovalóide corresponde às medidas de Markov com mesma entropia. Assim, é possível agora descrever como a dinâmica muda ao variarmos a medida de Markov ao longo de uma linha principal de algum ovalóide, com entropia $\lambda$ escolhida entre $\log \Phi$ e $\log 2$. Estudos preliminares indicam uma distribuição complexa de linhas principais nestas superfícies com seis singularidades, os pontos umbílicos. 


\section{Agradecimentos}

Os dois últimos autores agradecem o suporte técnico da Coordenação do Curso Interdisciplinar em Ciência e Tecnologia (BiCT/UFMA, São Luís/MA) durante a realização do Trabalho de Conclusão de Curso que deu origem a este artigo, sob orientação do primeiro autor. Os autores agradecem também ao Prof. Dr. José Antônio Pires Ferreira Marão pela leitura cuidadosa do manuscrito original e por valorosas contribuições ao texto, o que melhorou substancialmente sua apresentação.

\section{Referências}

APOSTOL, Tom M. Cálculo. v. 2, 1. ed. Barcelona: Editorial Reverté, 1981.

CARMO, M. P. do. Geometria diferencial de curvas e superfícies. 4. ed. Rio de Janeiro: SBM, 2010.

LEIGHTON, Walter. On Liapunov functions with a single critical point. Pacific Journal of Mathematics, v. 19, n. 3, p. 467-472, 1966. DOI: http://dx.doi.org/10.2140/pjm.1966.19.467.

MARKOWSKY, G. Misconceptions about the Golden Ratio. The College Mathematics Journal, v. 23, n. 1, p. 2-19, 1992. DOI: https://doi.org/10.1080/07468342.1992.11973428.

MILNOR, J. Morse Theory. Princeton: Princeton University Press, 1973.

MUMFORD, D.; SERIES, C.; WRIGHT, D. Indra's Pearl: The Vision of Felix Klein. Cambridge: Cambridge University Press, 2002.

O’NEILL, Barret. Elementary Differential Geometry. 2. ed. rev. Elsevier, 2006.

RUDIN, W. Principles of Mathematical Analysis. International Series in Pure and Applied Mathematics. 3. ed. McGraw-Hill, 1976.

VIEIRA, A.; MANDELA, L.; FERNANDES, P.; MOURA, V. Entropia máxima em inversões geométricas. REMAT: Revista Eletrônica da Matemática, Bento Gonçalves, v. 4, n. 1, p. 174-183, ago. 2018. DOI: https://doi.org/10.35819/remat2018v4i1id2585.

VIEIRA, A.; MANDELA, L.; FERNANDES, P.; MOURA, V. Geometria plana, cadeia de Markov e caos. Revista Eletrônica Paulista de Matemática, Bauru, v. 11, p. 34-47, dez. 2017. DOI: https://doi.org/10.21167/cqdvol11201723169664avlmpf vm3447. 